\title{
Experimental preparation of Werner state via spontaneous parametric down-conversion
}

\author{
Yong-Sheng Zhangt, Yun-Feng Huang, Chuan-Feng Li, and Guang-Can Guot \\ Laboratory of Quantum Information, University of Science and Technology of \\ China, CAS, Hefei 230026, People's Republic of China
}

\begin{abstract}
We present an experiment of preparing Werner state via spontaneous parametric down-conversion and controlled decoherence of photons in this paper. In this experiment two independent BBO (beta-barium borate) crystals are used to produce down-conversion light beams, which are mixed to prepare Werner state.

PACS number(s): 03.65.Ud, 42.50.-p, 03.67.-a
\end{abstract}

\section{INTRODUCTION}

Entanglement is not only one of the most striking features of quantum mechanics, it also plays a crucial role in the field of quantum information [1,2, 3, 3,5]. Entangled states are important resources for most applications of quantum information such as quantum key distribution [3], superdense coding [6], quantum teleportation [5] and quantum error correction [7] etc. Although the best performance of such tasks requires maximally entangled states (Bell states), the decoherence effects due to the environment make the pure entangled state into a statistical mixture and degrade quantum entanglement in the real word. For a practical purpose, a purification scheme may be applied to the degraded entanglement 8.9.

One of the most important degraded Bell states is Werner state [10], A Werner state in $2 \times 2$ system takes the following form $[8]$ :

$$
\rho_{W}=\frac{1-F}{3} I_{4}+\frac{4 F-1}{3}\left|\Psi^{-}\right\rangle\left\langle\Psi^{-}\right| .
$$

where $I_{n}$ denotes the $n \times n$ identity matrix and $\left|\Psi^{-}\right\rangle$is the singlet state of the four Bell states

$$
\begin{aligned}
& \left|\Phi^{ \pm}\right\rangle=\frac{1}{\sqrt{2}}(|00\rangle \pm|11\rangle), \\
& \left|\Psi^{ \pm}\right\rangle=\frac{1}{\sqrt{2}}(|01\rangle \pm|10\rangle) .
\end{aligned}
$$

The Werner state $\rho_{W}$ is characterized by a single real parameter $F$ called fidelity. This quantity measures the overlap of Werner state with a Bell state. In the case where $F \leqslant 1 / 2$, the state is separable, and thus has no entanglement to recover or maintain. And a Werner state with $F>(2+3 \sqrt{2}) / 8 \approx 0.78$ violates the Clauser-Horne-Shimony-Holt (CHSH) inequality [11, 12, 13].

There are many theoretical investigations on Werner state 14, and the Werner state plays an important role in entanglement purification [8], nonlocality [10], entanglement measure [15] and etc. However, there is no report of experimental realization of Werner state to date. At present, the most accessible and controllable source of entanglement is obtained from the process of spontaneous parametric down-conversion in a nonlinear crystal. In this paper, we present an experimental preparation of bi-photon Werner state via spontaneous parametric down-conversion and the prepared state is in the form

$$
\rho_{W}^{\prime}=x\left|\Phi^{-}\right\rangle\left\langle\Phi^{-}\right|+(1-x) \frac{I_{4}}{4},
$$

which can be transformed to $\rho_{W}$ by a local unitary transformation of $\sigma_{x} \otimes I$, where $\sigma_{x}$ is one of Pauli operators and $I$ is the identity operator.

*Electronic address: yshzhang@ustc.edu.cn

${ }^{\dagger}$ Electronic address: gcguo@ustc.edu.cn 
There are previous works on preparing mixed single photon or bi-photon state like in Ref. [16,20,21,22]. A. G. White et al. 20 have reported an optical two-qubit source that can produce a wide range of states in the form

$$
\rho=x\left|\Phi^{+}\right\rangle\left\langle\Phi^{+}|+(1-x)| 01\right\rangle\langle 01| .
$$

C. Zhang 22 has proposed a theoretical protocol to produce an arbitrary two-bit mixed state by using beam splitters with variable polarization transmission coefficients and single-mode optical fibers. However, in this paper, we propose a different way to prepare Werner states conveniently in experiment.

This paper is organized as follows. In Sec. II, the experimental set-up is described. The experimental results is given in Sec. III and the discussion and summary is given in Sec. IV.

\section{EXPERIMENTAL SET-UP}

In our experiment, the Werner state is bi-photon's polarization state, which is obtained by mixing a complete mixed state and an entangled state.

The experimental set-up is depicted in Fig. 1. The 1.7-mm-diam pump beam at $351.1 \mathrm{~nm}$ (single line, $100 \mathrm{~mW}$ ) is produced by an argon ion laser (Coherent, Sabre, model DBW25/7), and directed to the first BBO (beta-barium borate) crystal (cut for type-I phase matching, optic axis cut at $\theta=35.0^{\circ}, 1 \mathrm{~mm}$ thick) after passing through a polarizing beam splitter (PBS) to give a pure horizontal polarization state. Two entangled photon beams (polarized in vertical direction) produced by the BBO crystal passing through a sequence of quartz plates, with an optic axis set in the diagonal direction. It is pointed out that the photon polarization state will decohere in such an environment 116. The thickness of quartz plates is set in such a way that the photon's polarization decoheres completely (See Ref. [27], the difference between optical path of horizontal and vertical optical polarizations in the quartz plates is $153 \lambda_{0}$, and $\lambda_{0}$ is the central wave length of the down-converted light.). The remained pump beam passes through the BBO crystal and is rotated to $\frac{1}{\sqrt{2}}(|H\rangle+|V\rangle)$ by a half-wave plate (HWP) and is transmitted through the second BBO crystal which is the same as that proposed by Kwiat in Ref. [17.18 to produce polarization-entangled photons in such a state. The down-converted light beams produced by the first BBO were reflected by four reflectors and passed through the second BBO crystal to be mixed with the down-converted beams produced in the second BBO. Note the reason that the pump was set in single line instead of single frequency is to decrease the coherent length (about $4 \mathrm{~cm}$ in our experiment) of the pump light and avoid the interference between the down-converted light beams from the two BBO crystals.

\section{Figure 1.}

At the end, the bi-photon polarization state is measured tomgraphically 199 .18 by quarter-wave plates (QWP), HWP and PBS. We use 16 analyzer settings as listed in Table I. The photons are detected by using silicon avalanche photodiodes (EG\&G, SPCM-AQR) operated in the geiger mode. Each detector is preceded by a small iris (the diameter is $1.5 \mathrm{~mm}$ ) to define the spatial mode, a narrowband interference filter (IF) centered at $702 \mathrm{~nm}$ (Andover, 050FC46-25/7022 [23], full width at half is equal to $4.62 \mathrm{~nm}$ ) to reduce background and define the bandwidth of the photons, and a collection lens. The detector outputs are recorded in coincidence with a time-to-amplitude converter and a single-channel analyzer, leading to an effective coincidence window of $5 \mathrm{~ns}$. The resulting rate of accidental coincidences is less than $1 \mathrm{~s}^{-1}$, which can be neglected compared with the typical rate of the true coincidences, which is about $300 \mathrm{~s}^{-1}$.

We also test whether the states produced by this set-up violate the CHSH inequality. This inequality shows that $|S| \leq 2$ for any local realistic theory, where

$$
S=E\left(\theta_{1}, \theta_{2}\right)+E\left(\theta_{1}^{\prime}, \theta_{2}\right)+E\left(\theta_{1}, \theta_{2}^{\prime}\right)-E\left(\theta_{1}^{\prime}, \theta_{2}^{\prime}\right)
$$

and $E\left(\theta_{1}, \theta_{2}\right)$ is given by

$$
\frac{C\left(\theta_{1}, \theta_{2}\right)+C\left(\theta_{1}^{\perp}, \theta_{2}^{\perp}\right)-C\left(\theta_{1}^{\perp}, \theta_{2}\right)-C\left(\theta_{1}, \theta_{2}^{\perp}\right)}{C\left(\theta_{1}, \theta_{2}\right)+C\left(\theta_{1}^{\perp}, \theta_{2}^{\perp}\right)+C\left(\theta_{1}^{\perp}, \theta_{2}\right)+C\left(\theta_{1}, \theta_{2}^{\perp}\right)}
$$

and $C\left(\theta_{1}, \theta_{2}\right)$ is the coincidence rate of two detectors when the polarization analyzer angels are set in $\theta_{1}$ and $\theta_{2}$. In this experiment, we selected the settings: $\theta_{1}=-22.5^{\circ}, \theta_{1}^{\perp}=67.5^{\circ}, \theta_{1}^{\prime}=22.5^{\circ}, \theta_{1}^{\prime \perp}=112.5^{\circ}, \theta_{2}=0^{\circ}, \theta_{2}^{\perp}=90^{\circ}$, $\theta_{2}^{\prime}=45^{\circ}, \theta_{2}^{\prime \perp}=135^{\circ}$. 


\section{EXPERIMENTAL RESULTS}

We can adjust the intensity proportion (it can be tuned by adjusting the position of the reflectors) between the down-converted light from two BBO crystals to obtain Werner states with different coefficients $x$. Two output states have been produced in this experiment, for one of them the CHSH inequality is violated while the other is not.

The tomographic results are shown in Table I.

Table I.

From the data in the third column of Table I we can obtain the density matrix of the first output state directly, however, it is not non-negative definite [19]. We have used the maximum likelihood estimation [19] to construct a non-negative definite density matrix

$$
\rho_{1}=\left(\begin{array}{cccc}
0.4169 & 0.0203+0.0022 i & 0.0094-0.0237 i & -0.3476+0.0296 i \\
0.0203-0.0022 i & 0.0531 & -0.0122-0.0527 i & -0.0163+0.0005 i \\
0.0094+0.0237 i & -0.0122+0.0527 i & 0.0559 & -0.0134-0.0191 i \\
-0.3476-0.0296 i & -0.0163-0.0005 i & -0.0134+0.0191 i & 0.4741
\end{array}\right) .
$$

The fit Werner state

$$
\rho_{1}^{\prime}=x_{1}\left|\Phi^{-}\right\rangle\left\langle\Phi^{-}\right|+\left(1-x_{1}\right) \frac{I_{4}}{4}
$$

(where $\left.x_{1}=0.801 \pm 0.005\right)$ satisfies

$$
\begin{aligned}
F\left(\rho_{1}, \rho_{1}^{\prime}\right) & =\operatorname{Tr}^{2}\left(\sqrt{\rho_{1}^{\prime 1 / 2} \rho_{1} \rho_{1}^{\prime 1 / 2}}\right) \\
& =\max F\left(\rho_{1}, \rho_{W}\right),
\end{aligned}
$$

where $\rho_{W}$ is an arbitrary two-bit Werner state and the fidelity 24] $F\left(\rho_{1}, \rho_{1}^{\prime}\right)$ is equal to 0.932 in this experiment. The CHSH correlation value [Eq. (3)] $|S|=2.198 \pm 0.004>2$, (the uncertainty of the HWP is $\Delta \theta \simeq 0.2^{\circ}$ ) which violates the CHSH inequality. The theoretical value of $|S|$ of state $\rho_{1}^{\prime}$ is equal to 2.266.

The tomographic results of the second output are shown in the sixth column of Table I. From these data we can obtain the density matrix of the second output state

$$
\rho_{2}=\left(\begin{array}{cccc}
0.3949 & -0.0217+0.0398 i & -0.0080+0.0024 i & -0.1657+0.0150 i \\
-0.0217-0.0398 i & 0.1285 & 0.0165-0.0011 i & -0.0075+0.0504 i \\
-0.0080-0.0024 i & 0.0165+0.0011 i & 0.1311 & -0.0152+0.0228 i \\
-0.1657-0.0150 i & -0.0075-0.0504 i & -0.0152-0.0228 i & 0.3455
\end{array}\right)
$$

The fit Werner state is

$$
\rho_{2}^{\prime}=x_{2}\left|\Phi^{-}\right\rangle\left\langle\Phi^{-}\right|+\left(1-x_{2}\right) \frac{I_{4}}{4},
$$

where $x_{2}=0.405 \pm 0.005$ and the fidelity $F\left(\rho_{2}, \rho_{2}^{\prime}\right)$ is equal to 0.982 . The CHSH correlation value $|S|=1.380 \pm 0.008<$ 2 , which satisfies the CHSH inequality. The theoretical value of $|S|$ of state $\rho_{2}^{\prime}$ is equal to 1.146. We should note that there is significant difference in count rates between the two sets of results. In our experiment, the intensity proportion of down-converted light beams between two BBO crystals is tuned by adjusting the position of the reflectors. So the length of down-converted photon's optical path between the source and the detector has been changed for preparing the second state. Therefore, the count rates have been decreased significantly.

We can use the "linear entropy" to quantify the degree of mixture of a quantum state and use the tangle to measure the degree of entanglement of a two-bit quantum state [19,25]. The linear entropy for a two-bit system is defined by

$$
P=\frac{4}{3}\left(1-\operatorname{Tr}\left\{\rho^{2}\right\}\right)
$$

The tangle is defined as $T=C^{2}$, where $C$ is the concurrence in Ref. 25. In this experiment, for the first density matrix in Eq. (4), $P=0.46 \pm 0.03$ and $T=0.35 \pm 0.01$, and for the second density matrix in Eq. (7), $P=0.83 \pm 0.03$ and $T=0.01 \pm 0.00$. 


\section{DISCUSSION AND CONCLUSION}

We have prepared two typical Werner states in this experiment and measured the density matrix tomographically. Two independent BBO crystals are used to produce parametric down-conversion light beams which can be mixed to prepare a wide range of two-bit quantum states. In our experiment, the intensity proportion of down-converted light beams between two BBO crystals are tuned by adjusting the position of the reflectors. In fact, the proportion can be tuned with linear optical elements, which is shown in Fig. 2.

\section{Figure 2.}

Although the output light beams were produced by classically mixing (not quantum superposing) the downconverted light beams, it is hard (but not in principle) to distinguish from which crystals the output photon is produced since the pump light is continuous. However, if we use the pulse pump we can distinguish which crystal the output photon from by measuring the photon's arrival time, that is, we can distill the classical information of the mixed state and it can be regarded as a pure state.

We expect that this method can be modified to prepare arbitrary two-bit states conveniently and this source can be used in experimental investigation of entanglement purification or other quantum information tasks.

\section{ACKNOWLEDGMENTS}

This work was supported by the National Fundamental Research Program (2001CB309300), the National Natural Science Foundation of China, the Innovation Funds from Chinese Academy of Sciences (CAS), and was also funded by the outstanding $\mathrm{Ph}$. D. thesis award and the CAS's talented scientist award rewarded to Lu-Ming Duan.

\section{Appendix}

For a single photon, the non-dissipative coupling between photon frequency and polarization in a birefringent media leads to decoherence of polarization, provided we regard the freedom of frequency as "environment" [16,19,20].

The single-photon's initial state is described by a pure product state of polarization and frequency,

$$
|\Psi\rangle=\left(a_{1}|H\rangle+a_{2}|V\rangle\right) \otimes \int d \omega A(\omega)|\omega\rangle .
$$

with basis $|H\rangle$ (horizontal polarization) and $|V\rangle$ (vertical polarization) denoted by

$$
|H\rangle=\left(\begin{array}{l}
1 \\
0
\end{array}\right),|V\rangle=\left(\begin{array}{l}
0 \\
1
\end{array}\right)
$$

respectively, and $A(\omega)$ is the complex amplitude corresponding to $\omega$, normalized so that

$$
\int d \omega|A(\omega)|^{2}=1
$$

After being transmitted by the birefringent medium whose length is $x$, the final state of the photon is

$$
\left|\Psi^{\prime}(x)\right\rangle=a_{1}|H\rangle \otimes \int d \omega A(\omega) e^{-\frac{1}{2} i \omega\left(n_{H}-n_{V}\right) x / c}|\omega\rangle+a_{2}|V\rangle \otimes \int d \omega A(\omega) e^{\frac{1}{2} i \omega\left(n_{H}-n_{V}\right) x / c}|\omega\rangle .
$$

Where $n_{H}\left(n_{V}\right)$ is the refractive index of horizontal (vertical) polarization. It is necessary to note that although $n_{H}$ and $n_{V}$ are dependent on frequency $\omega$, the value of $n_{H}-n_{V}$ does not vary obviously with frequency varying in a small scale and we regard it as constant. The density operator of the polarization state can be obtained by tracing over frequency degrees of freedom from the complete density operator

$$
\rho(x)=\left(\begin{array}{ll}
a_{1} a_{1}^{*} & a_{1} a_{2}^{*} \Gamma(x) \\
a_{1}^{*} a_{2} \Gamma^{*}(x) & a_{2} a_{2}^{*}
\end{array}\right),
$$

where

$$
\Gamma(x)=\int d \omega A(\omega) A^{*}(\omega) e^{-i \omega\left(n_{H}-n_{V}\right) x / c} .
$$


From Eq. (A-3) it can be seen that the evolution of the polarization's states depend on the character of the "environment" which is described by the function $A(\omega)$.

The spectrum of the photon's frequency is defined by the narrowband interference filter (IF). In this experiment, the spectrum of the photon's frequency is rectangular function. To obtain this environment, we use an interference filter whose spectrum's shape is approximate to rectangle and the full width at half maximum (FWHM) is $\delta=4.62$ nm. (Andover, 050FC46-25/7022 [23]). The theoretical value of $\Gamma(x)$ is

$$
\Gamma(x)=\frac{2 i c}{x\left(n_{H}-n_{V}\right) \Delta \omega} e^{i \omega_{0} x\left(n_{H}-n_{V}\right) / c} \sin \frac{x\left(n_{H}-n_{V}\right) \Delta \omega}{2 c}
$$

and

$$
|\Gamma(x)|=\frac{2 c}{x\left(n_{H}-n_{V}\right) \Delta \omega}\left|\sin \frac{x\left(n_{H}-n_{V}\right) \Delta \omega}{2 c}\right|,
$$

where we assume that $n_{H}>n_{V}$.

Figure 3.

In the experiment 27 (See Fig. 3, the BBO crystal in the figure is cut at a degenerate type-I phase matching angle), we first measure the density matrix of the decohered photon's polarization tomgraphically [19]:

$$
\rho^{\prime}=\left(\begin{array}{ll}
a_{1} & a_{2} \\
a_{2}^{*} & a_{3}
\end{array}\right) .
$$

The experimental value of $|\Gamma(x)|$ can be calculated as

$$
|\Gamma(x)|=\left|a_{2} / \sqrt{a_{1} a_{3}}\right| .
$$

The results are shown in Fig. 4. The coherence of the photon's polarization degenerates as theoretical expected.. It is interesting that the coherence is recovered to some extent after it degenerated completely, which is similar to the Fraunhofer diffraction at a rectangular aperture (or slit) [26]. However, since the spectrum of the filter is not strictly rectangular, there are some deviations at the tail of curve.

\section{Figure 4.}

[1] P. W. Shor, in Proceedings of the 35th Annual Symposium on the Foundations of Computer Science, p. 124-133 (IEEE Computer Society Press, Los Alamitos, California, 1984).

[2] C. H. Bennett and G. Brassard, in Proceedings of the IEEE International Conference on Computers, Systems, and Signal Processing, Bangalore, India, 1984 (IEEE, New York, 1984), p. 175.

[3] A. K. Ekert, Phys. Rev. Lett. 67, 661 (1991).

[4] A. Einstein, B. Podolsky and N. Rosen, Phys. Rev. 47, 777 (1935).

[5] C. H. Bennett, G. Brassard, C. Crépeau, R. Jozsa, A. Peres and W. K. Wootters, Phys. Rev. Lett. 70, 1895 (1993).

[6] C. H. Bennett and S. J. Wiesner, Phys. Rev. Lett. 69, 2881 (1992).

[7] D. Gottesman, e-print quant-ph/0004072.

[8] C. H. Bennett, G. Brassard, S. Popescu, B. Schumacher, J. A. Smolin and W. K. Wootters, Phys. Rev. Lett. 76, 722 (1996).

[9] D. Deutsch, A. Ekert, R. Jozsa, C. Macchiavello, S. Popescu and A. Sanpera, Phys. Rev. Lett. 77, 2818 (1996).

[10] R. F. Werner, Phys. Rev. A 40, 4277 (1989).

[11] J. F. Clauser, M. A. Horne, A. Shimony and R. A. Holt, Phys. Rev. Lett. 23, 880 (1969).

[12] S. Popescu, Phys. Rev. Lett. 72, 797 (1994).

[13] P. K. Aravind, Phys. Lett. A 200, 345 (1995); R. Horodecki, P. Horodecki, and M. Horodecki, ibid. 200, 340 (1995).

[14] J. Lee and M. S. Kim, Phys. Rev. Lett. 84, 4236 (2000); T. Hiroshima and S. Ishizaka, Phys. Rev A 62, 044302 (2000); A. O. Pittenger and M. H. Rubin, e-print quant-ph/0001110 (2000); S. Bose and V. Vedral, Phys. Rev. A 61, 040101(R) (2000); S. Ishizaka and T. Hiroshima, Phys. Rev. A 62, 022310 (2000); A. Felicetti, S. Mancini, and P. Tombesi, Phys. Rev. A 65, 062107 (2002). 
[15] P. W. Shor, J. A. Smolin, and B. M. Terhal, Phys. Rev. Lett. 86, 2681 (2001).

[16] P. G. Kwiat, A. J. Berglund, J. Altepeter, and A. G. White, Science 290, 498 (2000); A. J. Berglumd, e-print quant$\mathrm{p} / \mathrm{/0010001.}$.

[17] P. G. Kwiat, E. Waks, A. G. White, I. Appelbaum, and P. H. Eberhard, Phys. Rev A 60, R773 (1999).

[18] A. G. White, D. F. V. James, P. H. Eberhard, and P. G. Kwiat, Phys. Rev. Lett. 83, 3103 (1999).

[19] D. F. V. James, P. G. Kwiat, W. J. Munro, and A. G. White, Phys. Rev. A 64, 052312 (2001).

[20] A. G. White, D. F. V. James, W. J. Munro, and P. G. Kwiat, Phys. Rev. A 65, 012301 (2002).

[21] R. T. Thew and W. J. Munro, Phys. Rev. A 64, 022320 (2001).

[22] C. Zhang, e-print quant-ph/0107145.

[23] Part number: 050FC46-25/7022, http://www.andcorp.com.

[24] R. Jozsa, J. Mod. Opt. 41, 2315 (1994).

[25] W. K. Wootters, Phys. Rev. Lett. 80, 2245 (1998).

[26] M. Born and E. Wolf, Principles of Optics (7th edition), (Press of University of Cambridge, Cambridge, England, 1999).

[27] Y.-S. Zhang, Y.-F. Huang, C.-F. Li, and G.-C. Guo, unpublished.

Figure caption

Figure 1. Experimental set-up to produce bi-photon Werner state.

Figure 2. Experimental set-up to tune the intensity proportion of down-conversion light beams from two BBO crystals.

Figure 3. Experimental setup of controlled decoherence of single photon's polarization. The $351.1 \mathrm{~nm}$ line of an argon ion laser (Coherent, Sabre) is used to pump a BBO crystal (3 $\mathrm{mm}$ thick), which is cut at a degenerate type-I phase matching angle to produce a pair of entangled photons. Each photon of the entangled pair is polarized in state $|H\rangle$. Then the polarization of one photon of the entangled pair is rotated to $\frac{1}{\sqrt{2}}(|H\rangle+|V\rangle)$ by the half-wave plate (HWP). After decohering in the quartz plates, this photon's polarization state is measured tomographically by HWP, quarter-wave plate (QWP) and polarization beam splitter (PBS). The other photon of the pair is used to do coincidence detection. We measure the decohered photon's polarization with 4 analysis settings $(|H\rangle,|V\rangle,|H\rangle+|V\rangle,|H\rangle+i|V\rangle)$, allowing reconstruction of the density matrix.

Figure 4. Experimental results vs theoretical curve. The FWHM of the IF is $4.62 \mathrm{~nm}$ and the spectrum of the frequency is assumed to be rectangular. $x$ is the optical path difference between horizontal and vertical polarizations and $\lambda_{0}$ is the central wavelength - 702.2nm - of photons produced by SPDC.

Table I. Settings for measuring bi-photon Stokes parameters. $H, V$, and $D$ are horizontal, vertical, and diagonal $\left(45^{\circ}\right)$ linear polarization, respectively. $R$ is right circular polarization. The data are for state $\rho_{1}$ (counted over $100 \mathrm{~s}$ ).

Table II. The data are for state $\rho_{2}$ (counted over $100 \mathrm{~s}$ ). 


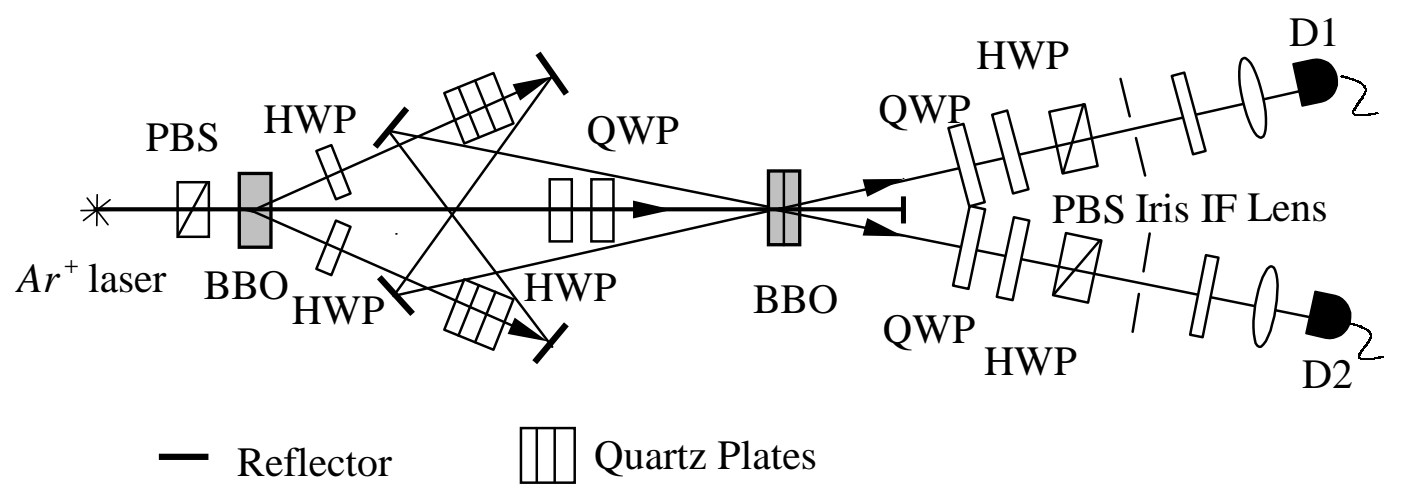

Figure 1 


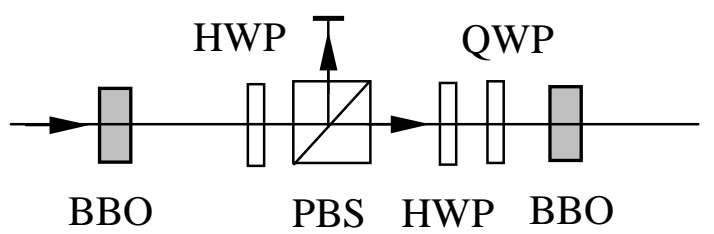

Figure 2 


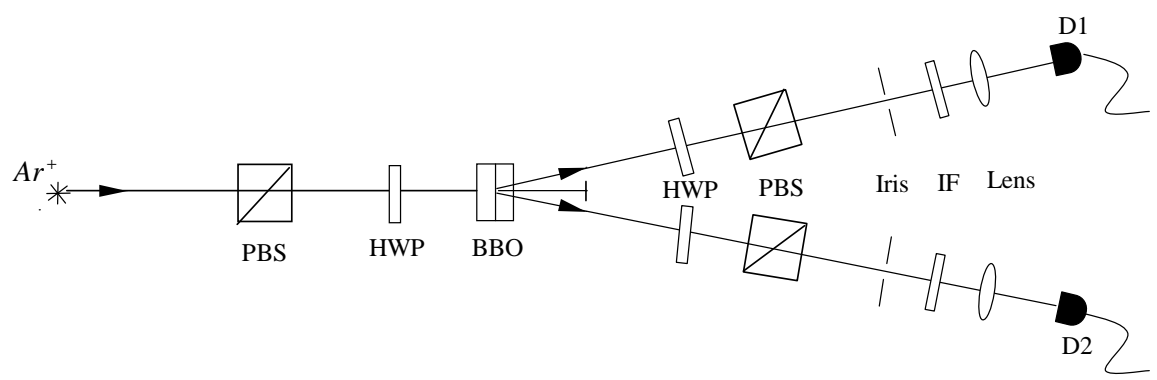

Figure 3. Experiment Setup 


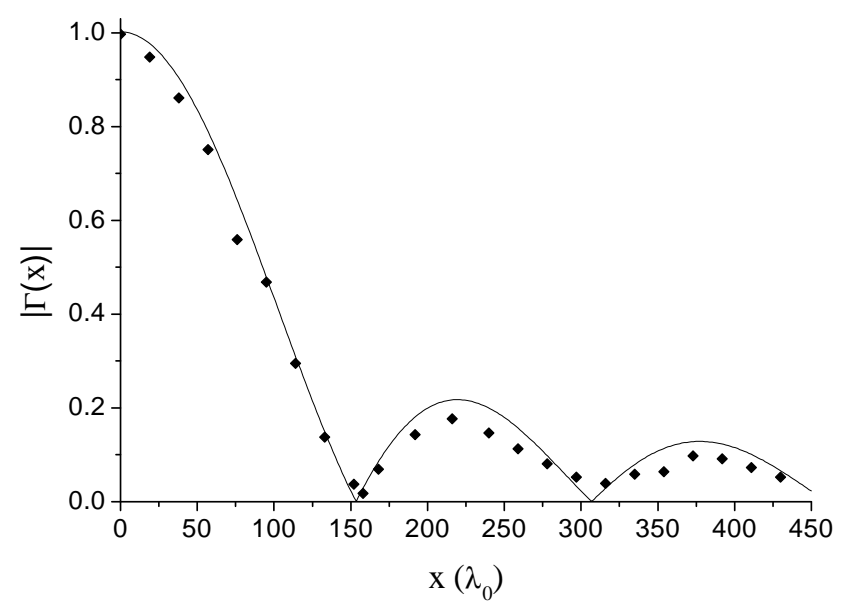

Fig. 4 


\begin{tabular}{ccrccc}
\hline $\begin{array}{c}\text { Analyzer } \\
\text { in arm 1 }\end{array}$ & $\begin{array}{c}\text { Analyzer } \\
\text { in arm 2 }\end{array}$ & $\begin{array}{c}\text { Coinc. } \\
\text { count }\end{array}$ & $\begin{array}{c}\text { Analyzer } \\
\text { in arm 1 }\end{array}$ & $\begin{array}{c}\text { Analyzer } \\
\text { in arm 2 }\end{array}$ & $\begin{array}{c}\text { Coinc. } \\
\text { count }\end{array}$ \\
\hline$H$ & $H$ & 31369 & $H$ & $H$ & 10136 \\
$H$ & $V$ & 3945 & $H$ & $V$ & 3298 \\
$H$ & $D$ & 18528 & $H$ & $D$ & 6159 \\
$H$ & $L$ & 18090 & $H$ & $R$ & 5695 \\
$V$ & $H$ & 4180 & $V$ & $H$ & 3365 \\
$V$ & $V$ & 35625 & $V$ & $V$ & 8867 \\
$V$ & $D$ & 18312 & $V$ & $D$ & 5725 \\
$V$ & $L$ & 18557 & $V$ & $R$ & 5532 \\
$D$ & $H$ & 17745 & $D$ & $H$ & 6546 \\
$D$ & $V$ & 17872 & $D$ & $V$ & 5891 \\
$D$ & $D$ & 5257 & $D$ & $D$ & 3829 \\
$D$ & $L$ & 21335 & $D$ & $R$ & 5210 \\
$R$ & $H$ & 19677 & $R$ & $H$ & 6688 \\
$R$ & $V$ & 20088 & $R$ & $V$ & 4788 \\
$R$ & $D$ & 20565 & $R$ & $D$ & 5085 \\
$R$ & $L$ & 5930 & $R$ & $R$ & 7272 \\
\hline
\end{tabular}

Table I 